\title{
Bump-on-tail instability across coupling and interaction-range regimes
}

\author{
Joseph J. Williams $\odot,{ }^{1}$ Gautham Dharuman, ${ }^{2,}{ }^{*}$ Mathieu Marciante, ${ }^{3, \dagger}$ James Hamilton Cooley, ${ }^{3}$ and Michael S. Murillo $\odot^{2}$ \\ ${ }^{1}$ Department of Aerospace Engineering and Department of Mathematics, University of Texas, Austin, Texas 78705, USA \\ ${ }^{2}$ Department of Computational Mathematics, Science and Engineering, Michigan State University, East Lansing, Michigan 48824, USA \\ ${ }^{3}$ Computational Physics and Methods Group, Los Alamos National Laboratory, Los Alamos, New Mexico 87545, USA
}

(Received 24 September 2018; revised manuscript received 11 July 2019; published 10 December 2019)

\begin{abstract}
The impact of collisionality and the range of the interparticle interaction on the bump-on-tail instability is examined both computationally and theoretically. Using three-dimensional, nonequilibrium molecular dynamics with a force law that varies continuously from long range (pure $1 / r$ Coulomb limit) to short range and across coupling regimes from weakly to strongly collisional, we examine properties of the instability; unlike other computational methods, molecular dynamics includes large-angle scattering, including the extreme limit of caging, and dynamical nonlinear screening across length scales as small as the interparticle spacing. A simpler theoretical model is also developed for comparison to the molecular dynamics results; we find that the two methods agree well in the appropriate limit, revealing that molecular dynamics is an accurate tool for the exploration of plasma instabilities. Our results reveal that the bump-on-tail instability exists only for long-range interactions.
\end{abstract}

DOI: 10.1103/PhysRevResearch.1.033166

\section{INTRODUCTION}

Kinetic and fluid instabilities are distinguishing features of most plasmas, including those that appear in the solar wind [1], inertial-confinement fusion experiments [2], material processing [3], neutron-star magnetospheres [4], earth's foreshock region [5,6], and tokamaks [7], among many other examples. A common type of instability is that of energy conversion from a directed current into random thermal motion; examples of this type of instability are the two-stream and bump-on-tail (BOT) instabilities. Early work on this class of instabilities was carried out by Bunemen, who examined electron currents colliding with initially stationary ions [8,9] and found rapid clustering of ions that destroyed these currents within a few plasma periods. Because these instabilities occur in a wide range of plasmas, including those with various levels of collisionality and containing various species, it is important to examine in detail the role of different types of interparticle interactions and varying levels of collisionality and interparticle correlations.

All plasmas exhibit some degree of collisionality, which, for simplicity, is often neglected in theoretical and computational models, yet instability growth is expected to be reduced in the presence of collisions. Early studies ad-

\footnotetext{
*Present address: Lawrence Livermore National Laboratory, Livermore, California 94550, USA.

${ }^{\dagger}$ Present address: CEA, DAM, DIF, 91297 Arpajon Cedex, France.

Published by the American Physical Society under the terms of the Creative Commons Attribution 4.0 International license. Further distribution of this work must maintain attribution to the author(s) and the published article's title, journal citation, and DOI.
}

dressed ion-ion collisions in the two-stream instability in thermonuclear plasmas using Krook models [10] and examined anomalous absorption of radiation energy by electrons using Fokker-Planck models [11]. In their seminal work, Takizuka and Abe [12] developed a method for including collisions in plasma simulations. The most natural computational method for describing collisional plasmas is molecular dynamics (MD), which inherently and self-consistently includes dynamical many-body correlations beyond the mean field and large-angle collisions. While MD has been used to examine phenomena on the hydrodynamic scale [13-15], our goal here is to examine nonequilibrium plasma instabilities driven by long-range electric fields across coupling regimes.

Before discussing the MD model in Sec. III, we develop a simple theoretical model in the next section that includes a variable-range interaction potential between particles and a simple model for collisionality. Results from both MD and the theoretical model are then presented in Sec. IV, before we summarize our work in Sec. V.

\section{THEORETICAL MODEL}

We first consider a simple theoretical model that can reveal the approximate impacts of collisions and the range of the effective interparticle pair potential $u(r)$. Consider a single-species plasma in which particles have an effective charge of $Z e$ and interact through a Yukawa potential $u(r)=$ $Z^{2} e^{2} \exp (-r / \lambda) / r$; this potential describes the pure Coulomb case when $\lambda \rightarrow \infty$ and in general the screened ionic case with electronic screening with finite $\lambda$. The Yukawa model is generic: The values of $Z$ and $\lambda$ can be chosen to apply to a wide range of plasma types and environments. Using $u(r)$ for a cold plasma with a cold bump at stream speed $V_{s}$ and 

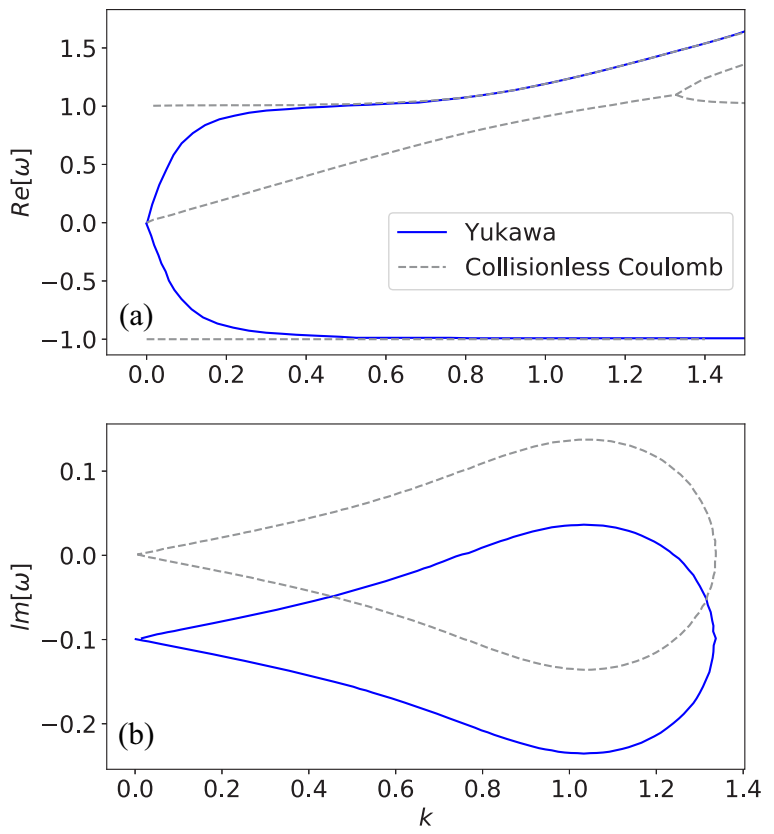

FIG. 1. Dispersion relation for $\Omega^{2}=0.01, V_{s}=1.0$, and two different combinations of $k_{r}$ and $v$, for the (a) real and (b) imaginary parts. The dashed lines correspond to the pure Coulomb collisionless case $\left(k_{r}=0.0\right.$ and $\left.v=0.0\right)$ and the solid blue lines correspond to a Yukawa interaction with $k_{r}=0.1$ and $v=0.1$. Adding collisionality shifts the imaginary curve downward, reducing the range of unstable $k$ values; adding screening $\left(k_{r} \neq 0\right)$ does not significantly affect the imaginary part, but creates an acoustic structure for the real part.

including a simple Krook model for collisions, we obtain the (dimensionless) dispersion relation

$$
\begin{gathered}
0=1-\frac{F(k)}{(\omega+i v)^{2}}-\frac{\Omega^{2} F(k)}{(\omega+i v-k)^{2}}, \\
F(k)=\frac{k^{2}}{k^{2}+k_{r}^{2}} .
\end{gathered}
$$
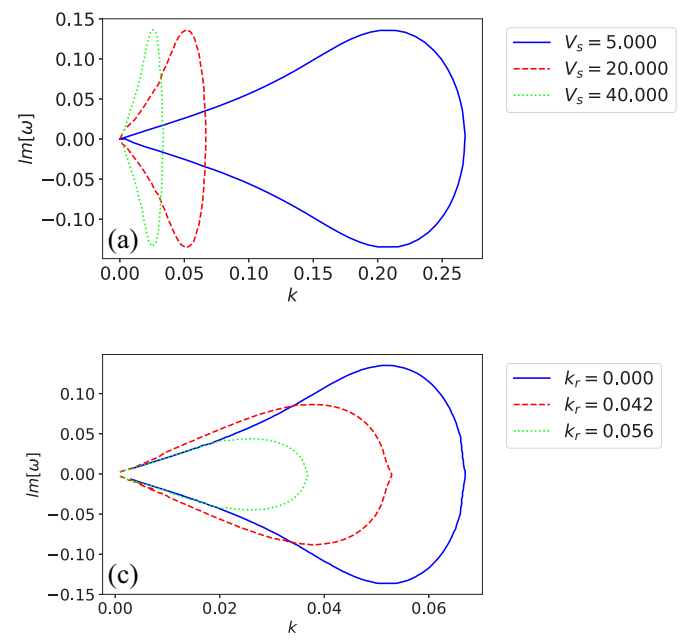

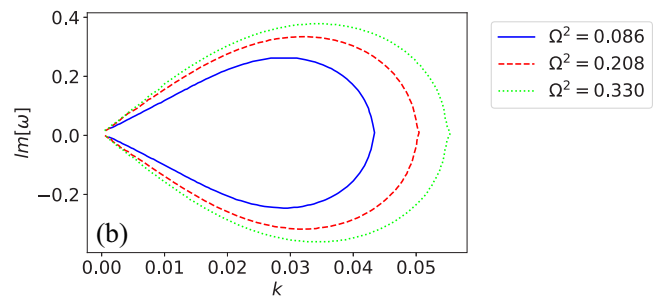

Here $\Omega=\omega_{s} / \omega_{b}$ is the ratio of stream to bulk plasma frequencies $\omega_{x}=\sqrt{4 \pi n_{x}\left(Z_{x} e\right)^{2} / m_{x}}$; assuming that the stream and bulk particles have the same charge and mass and occupy the same volume, this quantity reduces to $\Omega \sim \sqrt{n_{s} / n_{b}}$, which is usually a small quantity. The wave vector $k$ is in units of $\omega_{b} / V_{s}$ and $\omega$ is in units of $\omega_{b}$. The impact of the variablerange interaction enters through the factor $F(k)$ in (2), which arises from the Fourier transform of the Yukawa potential; the interaction range is characterized by the range parameter $k_{r}=$ $\frac{V_{s} \omega_{b}^{-1}}{\lambda}$, which is the ratio of the distance the stream moves in one plasma period to the range of the Yukawa potential. Note that $F(k)$ reduces to the pure Coulomb case when $k_{r} \rightarrow 0$ or $F(k)=1$. In this analysis, we have assumed that the screening factor is the same for both bulk and stream particles.

The solution of (1), a fourth-order polynomial, is readily found and is shown in Fig. 1. The base case is the set of gray dashed curves representing the collisionless Coulomb $(\lambda=\infty$ and $k_{r}=0$ ) result. In Fig. 1(a), which is the real part of the dispersion relation, we see the usual plasma oscillations at \pm 1 , as well as the acoustic branch that bifurcates at large $k$. The imaginary part is shown in Fig. 1(b); the top (gray dashed lines) set of curves for the imaginary part shows the well-known result that an instability $(\operatorname{Im}[\omega]>0)$ exists for a wide range of wave vectors. In contrast, the blue solid lines correspond to $v=0.1$ and $k_{r}=0.1$, which is the collisional screened case. Now, with a finite $k_{r}$, the real part of the dispersion is purely acoustic and the branches of $\operatorname{Im}[\omega]$ are shifted into the negative half plane by a constant shift of $v$, where the modes are now stable, except for a smaller region of instability in the range of about $0.75<k<1.25$.

Figure 2 shows detailed trends in $\operatorname{Im}[\omega]$ as each of the four main parameters is systematically varied, including the effects that variation in stream strength $\Omega$ [Fig. 2(b)], stream speed $V_{s}$ [Fig. 2(a)], range $k_{r}$ [Fig. 2(c)], and collisionality $v$ [Fig. 2(d)] have upon the dominant unstable mode $k_{\text {dom }}$. Figure 2(d) shows that increases in $v$ decrease $\operatorname{Im}[\omega]$ but do not affect $k_{\mathrm{dom}}$, suggesting that increasing the collisionality should suppress the growth of the instability but leave its mode unaffected. In Fig. 2(c) we see that increases in $k_{r}$ decrease

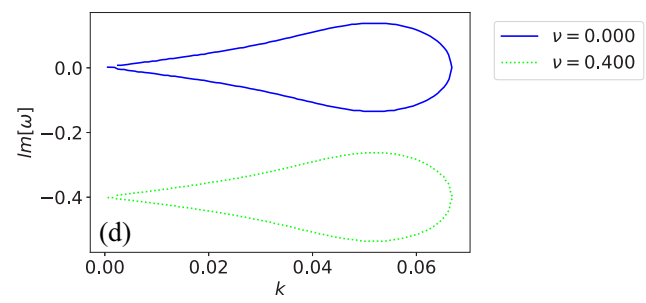

FIG. 2. Theoretical dispersion curves of $\operatorname{Im}[\omega(k)]$ for $V_{s}=40$ (top row) or $V_{s}=20$ (bottom row), $\Omega^{2}=0.11, k_{r}=0$, and $v=0$, except when that parameter is varied, as shown in the legends. 

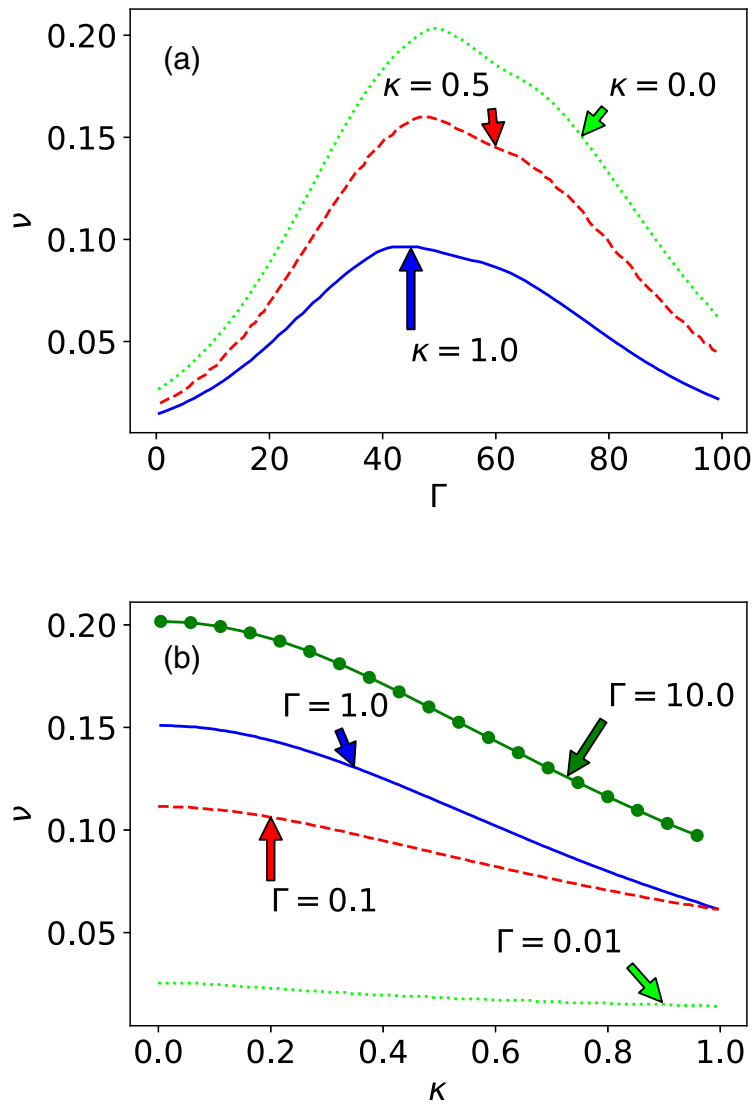

FIG. 3. Plot of $v$ as a function of (a) $\Gamma$ for $\kappa=0.0,0.5$, and 1.0 (top to bottom) and (b) $\kappa$ for $\Gamma=0.01,0.1,1.0$, and 10.0 (bottom to top).

both $k_{\mathrm{dom}}$ and $\operatorname{Im}[\omega]$, suggesting that increasing the range parameter both suppresses the growth of the instability and moves $k_{\mathrm{dom}}$ to longer wavelengths. Such predictions will be compared with our MD simulation results below.

Yukawa-based models are typically expressed in terms of two dimensionless parameters. Measuring distances in terms of the ion-sphere radius $a=(3 / 4 \pi n)^{1 / 3}$, a proxy for the interparticle spacing, and energies in units of the initial (bulk) temperature $T$ (in energy units where the Boltzmann constant $k_{B}=1$ ), the screening parameter $\kappa=a / \lambda$ and Coulomb coupling parameter $\Gamma=Z^{2} e^{2} / a T$ are often used, in particular when the coupling is large enough that discreteness of the particles plays a role. What is missing from this simple theoretical model is a prediction for the value of $v$, which is expected to have a strong dependence on $\kappa$ and $\Gamma$; however, Stanton and Murillo [16] have proposed a model for calculating $v(\Gamma, \kappa)$. Figure 3 shows $v$ as a function of $\Gamma$ [Fig. 3(a)] and of $\kappa$ [Fig. 3(b)]. The onset of caging occurs above approximately $\Gamma \sim 10$ [17]. Using this prescription for $v(\Gamma, \kappa)$, we can examine how the dominant unstable mode $k_{\text {dom }}$ and the growth rate $\operatorname{Im}\left[\omega\left(k_{\mathrm{dom}}\right)\right]$ of that mode vary and compare these results directly with the MD results.

\section{MOLECULAR DYNAMICS}

We now turn to our MD approach, which describes point particles at any length scale, allows for variable-range interac- tions that can vary continuously from long to short range (with the $1 / r$ Coulomb interaction as a limit), includes correlations in three dimensions, and allows the level of collisionality to be varied across orders of magnitude. With these constraints, we propose a MD model based on the Hamiltonian

$$
H=\sum_{i=1}^{N} \frac{p_{i}^{2}}{2 m}+\sum_{i<j} \frac{Z^{2} e^{2}}{r_{i j}} e^{-r_{i j} / \lambda}+U_{B},
$$

where all particles are assumed to have the same mass $m$ and charge $Z e .^{1}$ The number of particles in the simulation was chosen across a wide range both to ensure convergence and to control the aspect ratio of the periodic simulation cell for the desired wave vector; the typical number of particles was $N=10^{4}-10^{5}$. This model uses the Yukawa potential, which is described above. The term $U_{B}$ is a uniform background term that ensures the system is charge neutral; this term is needed to ensure convergence of the force sum in the pure Coulomb limit. The Yukawa interaction depends only on the relative distance $r_{i j}=\left|\mathbf{r}_{i}-\mathbf{r}_{j}\right|$ between the particles. Electric fields are manifestly included in this Hamiltonian through the Coulomb potential-energy terms, which satisfy a Poisson (or Helmholtz, for finite $\lambda$ ) equation. Relative to other computational approaches, such as particle-in-cell or Fokker-Planck models, MD describes waves and their damping fully consistently and for any level of collisionality. The only potential limitation of this approach is its limitation to small (in space and time) systems.

All MD simulations were performed using an efficient particle-particle-particle-mesh (PPPM) algorithm ${ }^{2}$ for screened Coulomb systems [18]; the PPPM algorithm naturally includes long-range electric fields. The simulations were carried out in three dimensions to correctly resolve the trajectories involved in many-body Coulomb collisions. Using a time step of $\Delta t=0.0025 \omega_{p}^{-1}$, where the plasma period is $\omega_{p}^{-1}=\left(4 \pi n Z^{2} e^{2} / m\right)^{-1 / 2}$, energy was well conserved for all cases, as confirmed by the lack of significant drift in the total energy using the error metric $\Delta E(\%)=\frac{100}{M} \sum_{j=1}^{M}\left|\frac{E\left(t_{j}\right)}{E(0)}-1\right|$, which is the percentage error accumulated over time relative to the initial energy of the system. For all simulations, $\Delta E(\%)<0.1 \%$. The Hamilton equations were integrated using the standard velocity Verlet algorithm. The degree of collisionality was controlled through the choice of initial temperatures, which were set through an equilibration phase with a Langevin thermostat at the target temperature, and the choice of the charge state $Z$.

The BOT initial condition was created using a three-step process. First, the target thermodynamic state was achieved through equilibration for $40 \omega_{p}^{-1}$ plasma periods, a value we

\footnotetext{
${ }^{1}$ Alternatively, the potential $(\sigma / r)^{n}$ also varies from Coulomb for $n=1$ to short range for large $n$ (the hard-sphere limit); we chose the exponential form because of its closer relation to plasma screening.

${ }^{2}$ For the PPPM algorithm, the Ewald parameter was varied over a range of about 1-10, in units of $a^{-1}$; for the particle-particle part, the cutoff radius was typically about $r_{c} \sim 5$ (in units of $a$ ); and for the particle-mesh part, the grid dimensions were $64 \times 64 \times 64$ and the $B$ splines were of order 6 . These parameters corresponded to an error of $\sim 10^{-6}\left(e^{2} / a_{i}^{2}\right)$ in the computed forces.
} 


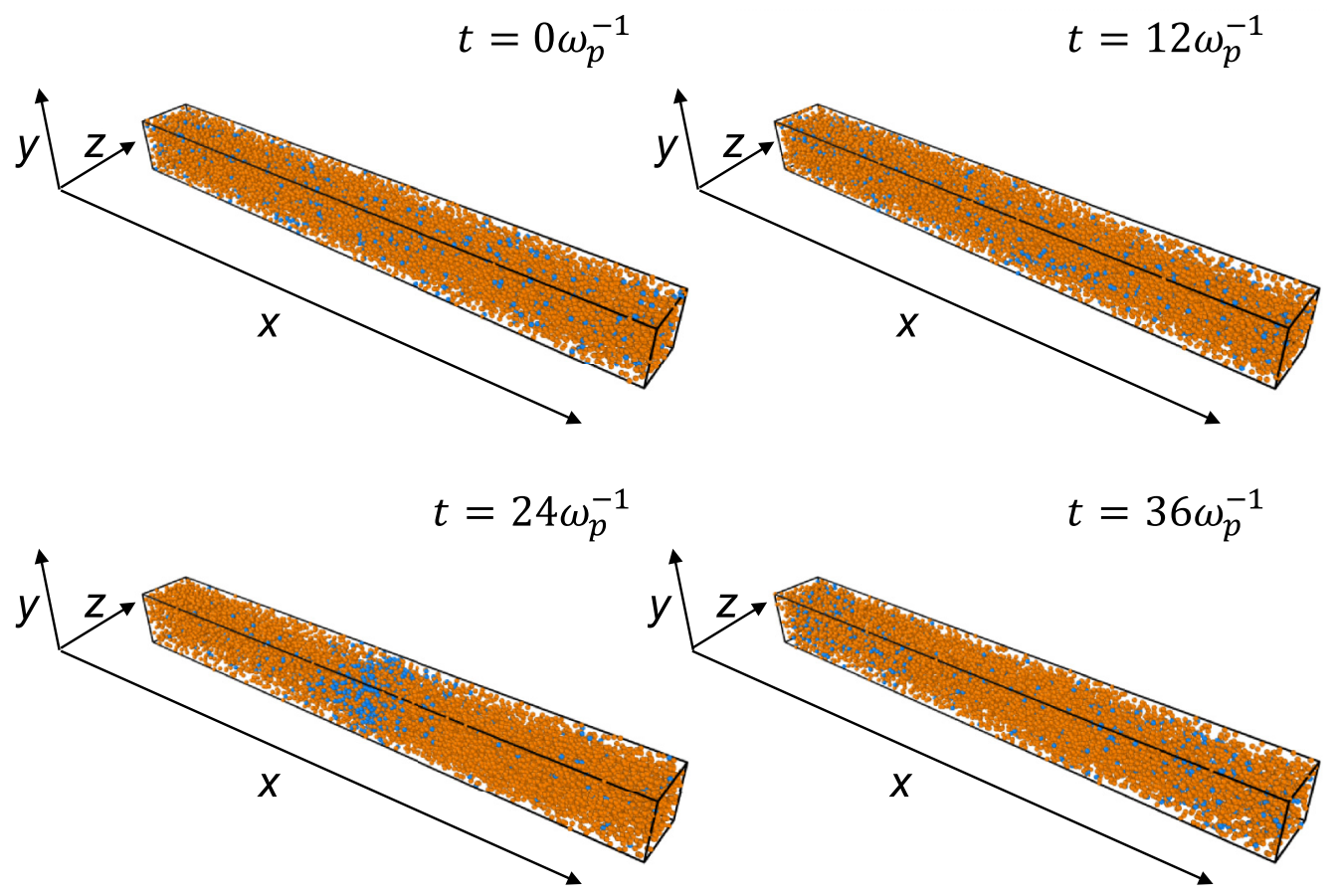

FIG. 4. Physical-space view of the individual particles in the MD simulation, at four times: $t=0 \omega_{p}^{-1}, 12 \omega_{p}^{-1}, 24 \omega_{p}^{-1}$, and $36 \omega_{p}^{-1}$. Initial bulk and stream particles are in orange (the majority of the particles) and blue (those particles that bunch in the cell center at $t=24 \omega_{p}^{-1}$ ), respectively. Molecular dynamics reveals the expected behavior of this instability.

determined empirically; results were very insensitive to longer equilibration periods. Second, another equilibration for $5 \omega_{p}^{-1}$ was carried out to establish an initial external density perturbation resulting from the form $U_{\mathrm{ext}}=p \cos (k x)$, where $p$ is the strength of the perturbation, $k=2 \pi m / L_{x}$, and $m$ is the mode of the perturbation. For this study, $m$ was varied to change which Fourier mode $k$ was seeded initially. The perturbation strength $p$ was also varied; $p$ was chosen to be small enough for the system to be in the linear regime, although, in principle, there is no restriction. In most cases, $p$ was chosen to be 0 to examine growth out of the natural noise in the system. At the end of this second equilibration phase, the third step of generating the BOT initial condition was to remove the external potential and the thermostat and add a bump to the velocity distribution by increasing the velocity of the specified number of particles in the simulation to the specified mean-stream velocity, with a distribution about the mean determined by the specified temperature. Finally, the main simulation was run and data were obtained for $40 \omega_{p}^{-1}$ plasma periods.

Figure 4 shows the physical-space evolution of the BOT instability obtained with MD; in the figure, each dot is a single charged particle, not a computational particle. In this example, there is no initial perturbation, and $V_{s}=40, c=0.1, \kappa=0$ (pure Coulomb), and $\Gamma=0.1$, where $c$ is the percentage of particles in the stream, $c=\frac{\Omega^{2}}{1+\Omega^{2}}$. A single, large, coherent, and long-lived longitudinal wave forms at approximately onequarter of the simulation time and dissipates by about threequarters of the simulation time; by the end of the simulation, the bulk and stream are well mixed, although remnants of the longitudinal wave are still visible.
The strength of the beam is characterized by the relative number of particles in the stream to that of the bulk, while in the cold-plasma dispersion relation, it is characterized by $\Omega^{2}$; these quantities are directly comparable. Taking into account the different dimensionless parameters of the two systems, the MD screening parameter $\kappa$ is exactly equal to $k_{r}$ in the dispersion relation. The MD parameters $\Gamma$ and $\kappa$ appear in the dimensionless theoretical collisionality $v$; here we employ the Stanton-Murillo [16] model. Each of these four parameters was systematically varied in both the theoretical model and the MD simulations, and the results of interest from this variation of parameters are the dominant wave vector of the instability $k_{\mathrm{dom}}$ and the growth rate of that instability $\operatorname{Im}[\omega]$.

\section{RESULTS}

In Fig. 5 we show the phase-space evolution of the BOT instability obtained using MD; each dot represents one particle. In this example, there is no initial perturbation $(p=0)$, $V_{s}=40, c=0.1, \kappa=0$ (pure Coulomb), and $\Gamma=0.1$ (weak coupling). In this case, there is a single, large, coherent, and long-lived phase-space vortex, forming at approximately onequarter of the simulation time and dissipating by about threequarters of the simulation time. By the end of the simulation, the bulk and stream are well mixed, although remnants of the vortex are still visible.

For these same conditions, the Fourier transform of the spatial density histogram is taken at each point in time. Then an exponential curve can be fit to the absolute value of the amplitude as a function of time to determine the growth rate of the instability; this process is shown in Fig. 6. Figure 7 shows 

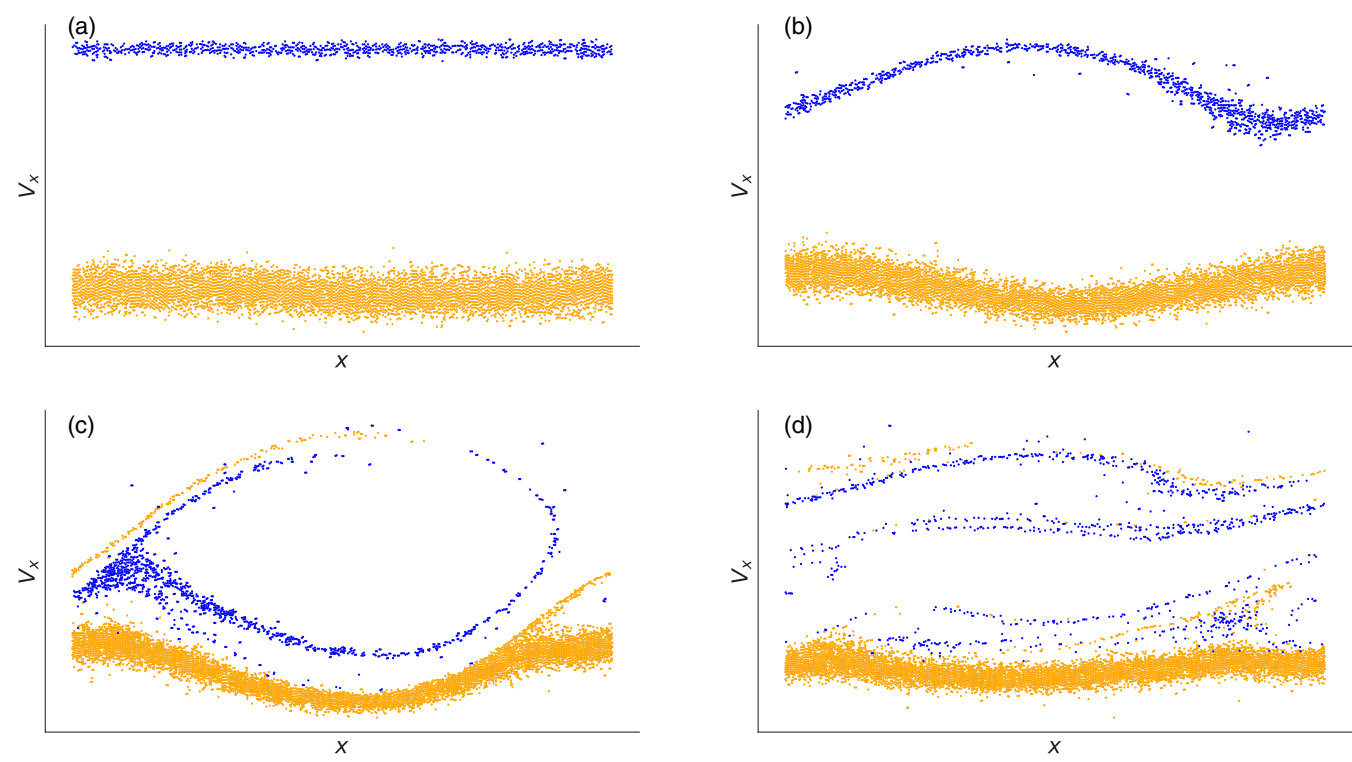

FIG. 5. Phase-space snapshots of the MD output at four times (a) $t=0 \omega_{p}^{-1}$, (b) $t=12 \omega_{p}^{-1}$, (c) $t=24 \omega_{p}^{-1}$, and (d) $t=36 \omega_{p}^{-1}$, where each dot is an individual particle. Initial bulk and stream particles are in yellow (lower points) and blue (upper points), respectively; note that the $\mathrm{MD}$ particles are in a $6 \mathrm{D}$ phase space, but we project onto a $2 \mathrm{D}$ phase space here for visualization purposes. The MD data reveal the expected behavior of the instability.

the growth rate from this fit is as a single data point, together with the dispersion relation generated from the theoretical model in the collisionless and collisional cases.

We move now to a systematic exploration of the full parameter space, varying the four main parameters of interest, each of which can be controlled and varied independently in
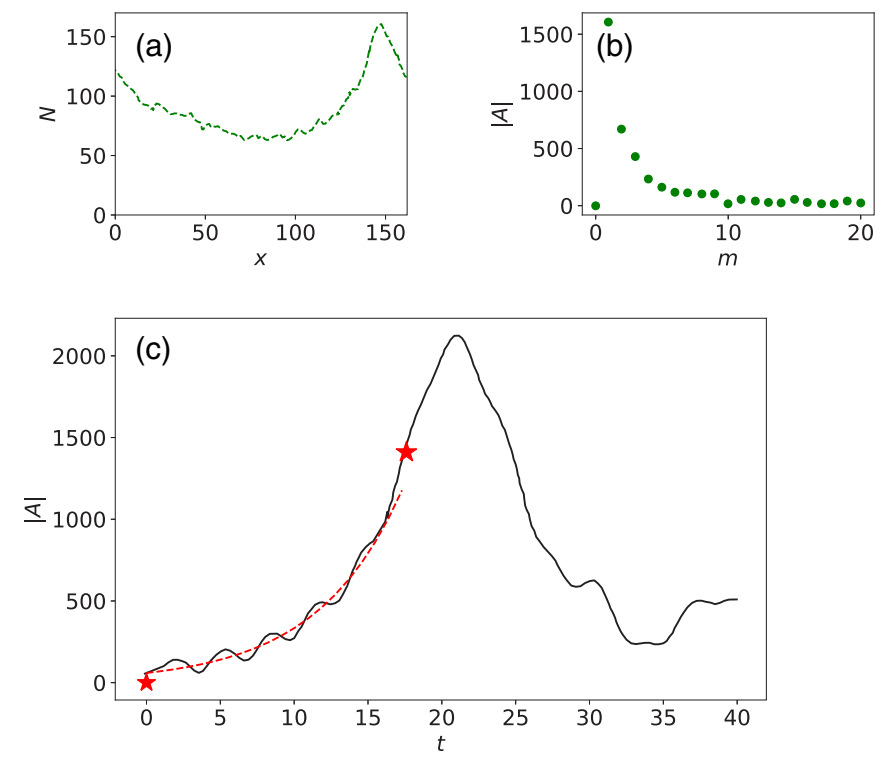

FIG. 6. Density of the system and its Fourier transform, for the same conditions as in Fig. 5. (a) Coordinate histogram, representing the particle density ( $N$ particles per bin in $x$ ) at $t=24 \omega_{p}^{-1}$, and (b) its Fourier transform. (c) Amplitude of the first mode of the system as a function of time, together with a fitted exponential growth curve; the early-time growth fits an exponential curve very well. The argument of this fitted exponential curve will be compared against the predicted dispersion curves in Fig. 7. both the theoretical model and MD simulations. These four main parameters in the theoretical model and the MD are the stream speed, the stream strength, the screening, and the collisionality. As variables in the MD system, they are $V_{s}, c$, $\kappa$, and $\Gamma$. As variables in the dispersion relation, they are $V_{s}$, $\Omega^{2}, k_{r}$, and $\nu$.

We focus on the dominant wave vector $k_{\text {dom }}$ of the instability and its growth rate $\operatorname{Im}[\omega]$, both initialized with $p=0$. Figures 8 and 9 show the effects that variation of any one of the four main parameters has upon $\operatorname{Im}[\omega]$ and $k_{\mathrm{dom}}$, respectively. The MD data points, shown in red in these figures, are

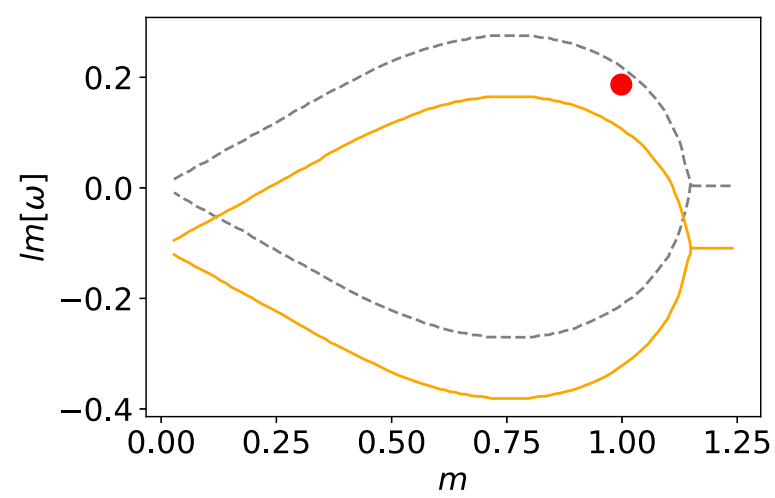

FIG. 7. Theoretical dispersion curves for $V_{s}=40, \Omega^{2}=0.11$, and $k_{r}=0$, compared with the growth rate calculated from the MD data (red circle). The dispersion curve calculated using $v=0$ is shown by the gray dashed line and the dispersion curve calculated using the calculated value of $v$ is shown by the orange solid line. The red point corresponds to the fitted growth rate obtained from $\mathrm{MD}$ and shown in Fig. 6. For this case, it is interesting to note that the MD result agrees better with the predicted dispersion curve for $v=0$ than the predicted dispersion curve for which $v$ is calculated, suggesting a weakness of the theoretical model. 

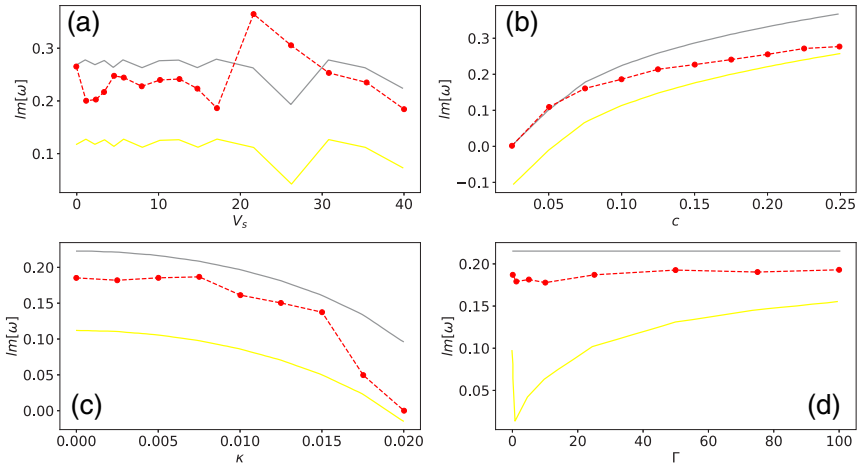

FIG. 8. Fitted MD growth rate (red dashed lines) compared with the predicted dispersion curves as individual parameters are varied. The gray solid (upper) curves again assume $v=0$, while the yellow solid (lower) curves use a calculated value of $v$. For the MD runs, $c=0.1, V_{s}=40, \kappa=0$, and $\Gamma=0.1$, except when one of these parameters was varied. The MD parameter values are shown on the lower axes and their theoretical equivalents are shown on the upper axes. As any one of the parameters is varied, we find that the MD growth rates stay within an envelope of predictions obtained from the dispersion relations, suggesting strong agreement between the theoretical model and the MD simulations.

each generated from unique runs in which a single parameter is varied while the other three parameters are held constant. Then a process identical to the one used to generate Fig. 6 is performed for each run to determine $k_{\text {dom }}$ and $\operatorname{Im}[\omega]$.

In Fig. 8, the gray solid (higher set) curves are generated from dispersion relations for which $v=0$, while the yellow solid (lower set) curves correspond to dispersion relations for which $v$ is calculated using the Stanton-Murillo model [16]. For almost all runs, the values of $\operatorname{Im}[\omega]$ calculated from the MD data fit neatly within the envelope formed using the predicted values for $v=0$ (gray curves) and using the calculated values for $v$ (yellow curves). The strong agreement between the numerical results and the predicted dispersion curves validate the use of our MD model for predicting growth rates of plasma instabilities. However, note that, in most cases, the MD tends to agree with the collisionless model. This behavior likely arises from weaknesses in the theoretical model and illustrates how MD can be used to validate standard plasma models. More work to address this finding is warranted.

\section{SUMMARY AND CONCLUSIONS}

In summary, this study investigated the BOT instability using MD thereby describing waves and their damping consistently for any level of collisionality. We found agreement
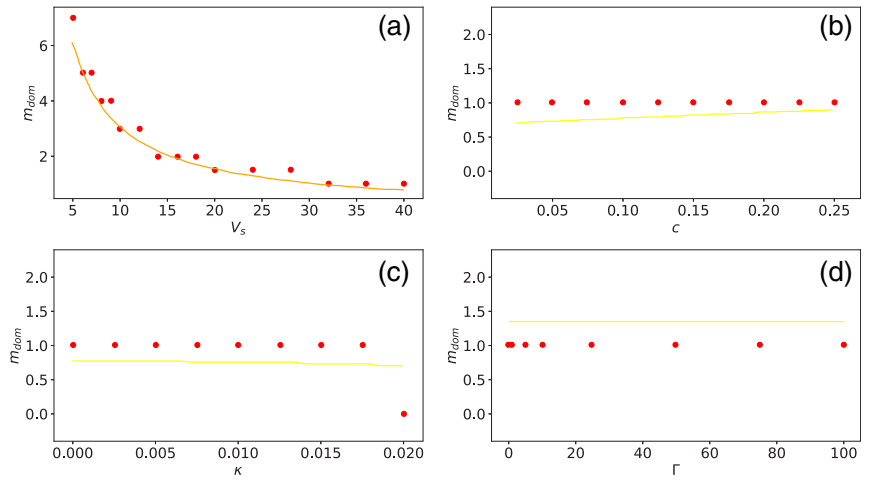

FIG. 9. Dominant mode of the instability in MD vs the four parameters (a) $V_{s}$, (b) $c$, (c) $\kappa$, and (d) $\Gamma$ that characterize the instability. Also shown as a solid line is the theoretical curve. As before, $c=0.1, V_{s}=40, \kappa=0$, and $\Gamma=0.1$, except when one of these parameters is varied. The MD parameter values are shown on the lower axes and their theoretical equivalents are shown on the upper axes. As any one of the parameters is varied, the dominant mode of the simulation generally agrees with the predictions of the dispersion relation, suggesting that the MD results predict the dominant mode of the instability accurately when compared with the theoretical dispersion relation.

between the predictions of the dispersion relation and the MD results. Specifically, the dominant mode of the instability was typically well aligned with that predicted by the dispersion relation, and the growth rate of the dominant modes fit within an envelope formed by one curve calculated using $v=0$ and another calculated using a calculated value of $v$. This work demonstrates that MD accurately describes nonequilibrium heterogeneous systems with long-range electric fields and does so with very high physics fidelity (e.g., dynamical manybody collisions). Thus, while MD is restricted to describing small systems in terms of absolute time and length scales, in terms of scaled variables, MD provides a validation approach for other methods traditionally employed for the study of large-scale plasma waves and instabilities.

Animations of our MD simulations are available in [19].

\section{ACKNOWLEDGMENTS}

M.S.M. and G.D. acknowledge the Air Force Office of Scientific Research for its support of this work under AFOSR Grant No. FA9550-17-1-0394. The authors thank Lisa Murillo for careful editing of the manuscript and Abudit Rai for his considerable help with the figures.
[1] P. H. Yoon, Kinetic instabilities in the solar wind driven by temperature anisotropies, Rev. Mod. Plasma Phys. 1, 4 (2017).

[2] R. Betti, V. N. Goncharov, R. L. McCrory, and C. P. Verdon, Growth rates of the ablative Rayleigh-Taylor instability in inertial confinement fusion, Phys. Plasmas 5, 1446 (1998).

[3] D. Sydorenko, I. D. Kaganovich, P. L. G. Ventzek, and L. Chen, Effect of collisions on the two-stream instability in a finite length plasma, Phys. Plasmas 23, 122119 (2016).
[4] A. K. Harding and D. Lai, Physics of strongly magnetized neutron stars, Rep. Prog. Phys. 69, 2631 (2006).

[5] A. J. Klimas, Trapping saturation of the bump-on-tail instability and electrostatic harmonic excitation in earth's foreshock, J. Geophys. Res.: Space 95, 14905 (1990).

[6] R. J. Fitzenreiter, A. J. Klimas, and J. D. Scudder, Detection of bump-on-tail reduced electron velocity distributions at the electron foreshock boundary, Geophys. Res. Lett. 11, 496 (1984). 
[7] V. S. Mukhovatov and V. D. Shafranov, Plasma equilibrium in a Tokamak, Nucl. Fusion 11, 605 (1971).

[8] O. Buneman, Instability, Turbulence, and Conductivity in Current-Carrying Plasma, Phys. Rev. Lett. 1, 8 (1958).

[9] O. Buneman, Dissipation of currents in ionized media, Phys. Rev. 115, 503 (1959).

[10] W. P. Gula and C. K. Chu, Effect of Krook model collisions on the two-stream instability, Phys. Fluids 16, 1135 (1973).

[11] C. E. Rathmann and J. Denavit, Simulation of collisional effects in plasmas, J. Comput. Phys. 18, 165 (1975).

[12] T. Takizuka and H. Abe, A binary collision model for plasma simulation with a particle code, J. Comput. Phys. 25, 205 (1977).

[13] M. Marciante and M. S. Murillo, Thermodynamic and Kinetic Properties of Shocks in Two-Dimensional Yukawa Systems, Phys. Rev. Lett. 118, 025001 (2017).
[14] K. Kadau, T. C. Germann, N. G. Hadjiconstantinou, P. S. Lomdahl, G. Dimonte, B. L. Holian, and B. J. Alder, Nanohydrodynamics simulations: An atomistic view of the RayleighTaylor instability, Proc. Natl. Acad. Sci. USA 101, 5851 (2004).

[15] D. Hirshfeld and D. C. Rapaport, Molecular Dynamics Simulation of Taylor-Couette Vortex Formation, Phys. Rev. Lett. 80, 5337 (1998).

[16] L. G. Stanton and M. S. Murillo, Unified description of linear screening in dense plasmas, Phys. Rev. E 91, 033104 (2015).

[17] Z. Donkó, G. J. Kalman, and K. I. Golden, Caging of Particles in One-Component Plasmas, Phys. Rev. Lett. 88, 225001 (2002).

[18] G. Dharuman, L. G. Stanton, J. N. Glosli, and M. S. Murillo, A generalized Ewald decomposition for screened Coulomb interactions, J. Chem. Phys. 146, 024112 (2017).

[19] See Supplemental Material at http://link.aps.org/supplemental/ 10.1103/PhysRevResearch.1.033166 for animations of our MD simulations. 